\title{
A Novel MYO6 Splice Site Mutation Causes Autosomal Dominant Sensorineural Hearing Loss Type DFNA22 with a Favourable Outcome after Cochlear Implantation
}

\author{
Alexander E. Volk ${ }^{a}$ Ruth Lang-Roth ${ }^{b}$ Goekhan Yigit ${ }^{c, d}$ Guntram Borck ${ }^{a}$ \\ Gudrun Nuernberg ${ }^{d, e}$ Stephan Rosenkranz ${ }^{f}$ Peter Nuernberg ${ }^{d, e}$ \\ Christian Kubisch $^{\text {a }}$ Dirk Beutner $^{\text {b }}$ \\ a Institute of Human Genetics, University of Ulm, Ulm, b Department of Otorhinolaryngology, Head and Neck \\ Surgery, ' Institute of Human Genetics, d Center for Molecular Medicine Cologne (CMMC), e Cologne Center for \\ Genomics (CCG), and ${ }^{\mathrm{f} C l i n i c}$ III for Internal Medicine, University of Cologne, Cologne, Germany
}

\section{Key Words}

Autosomal dominant hearing loss · DFNA22 - Myosin VI ·

Cochlear implantation

\begin{abstract}
Mutations in MYO6 encoding an atypical myosin motor protein important for inner ear hair cell function have been associated with autosomal recessive (DFNB37) and autosomal dominant (DFNA22) types of hearing loss in a few families worldwide. After genome-wide linkage analysis, we identified a novel MYO6 mutation at the splice acceptor site of exon 7 (c.554-1G >A) in an extended German family with autosomal dominant postlingual non-syndromic hearing impairment. Analysis of blood-derived cDNA revealed different aberrantly spliced mRNAs caused by the mutation, which are predicted to severely interfere with protein function. Two of the family members underwent cochlear implantation at ages 53 and 65. Here, we present detailed clinical data of this family which suggest a favourable outcome of cochlear implantation in hearing-impaired individuals with a MYO6 mutation.
\end{abstract}

Copyright $\odot 2013$ S. Karger AG, Basel (c) 2013 S. Karger AG, Basel

$1420-3030 / 13 / 0183-0192 \$ 38.00 / 0$

\section{Introduction}

Postlingual sensorineural hearing loss represents a heterogeneous group of sensory disorders of mostly multifactorial origin as for example seen in presbyacusis which accounts for the vast majority of cases of postlingual hearing impairment. However, in some families postlingual hearing loss is transmitted as a monogenic trait which in most cases shows an autosomal dominant inheritance pattern [Hilgert et al., 2009]. With recent advances in molecular genetics, our understanding of the pathogenesis of sensorineural hearing loss has greatly expanded and to date mutations in more than 60 genes involved in autosomal dominant or recessive non-syndromic hearing loss have been identified (see also http:// hereditaryhearingloss.org). Mutations in the gene encoding myosin VI (MYO6) are a relatively rare cause of autosomal dominant (DFNA22) and recessive (DFNB37) hearing loss [Ahmed et al., 2003; Hilgert et al., 2008; Melchionda et al., 2001; Mohiddin et al., 2004; Sanggaard et al., 2008] and pathogenic mutations have been published in 7 families worldwide. Together with MYO1A [Hasson

\section{KARGER}

E-Mail karger@karger.com

www.karger.com/aud
Alexander E. Volk, MD

Institute of Human Genetics, University of Ulm

Albert-Einstein-Allee 11

DE-89079 Ulm (Germany)

E-Mail alexander.volk@uni-ulm.de 


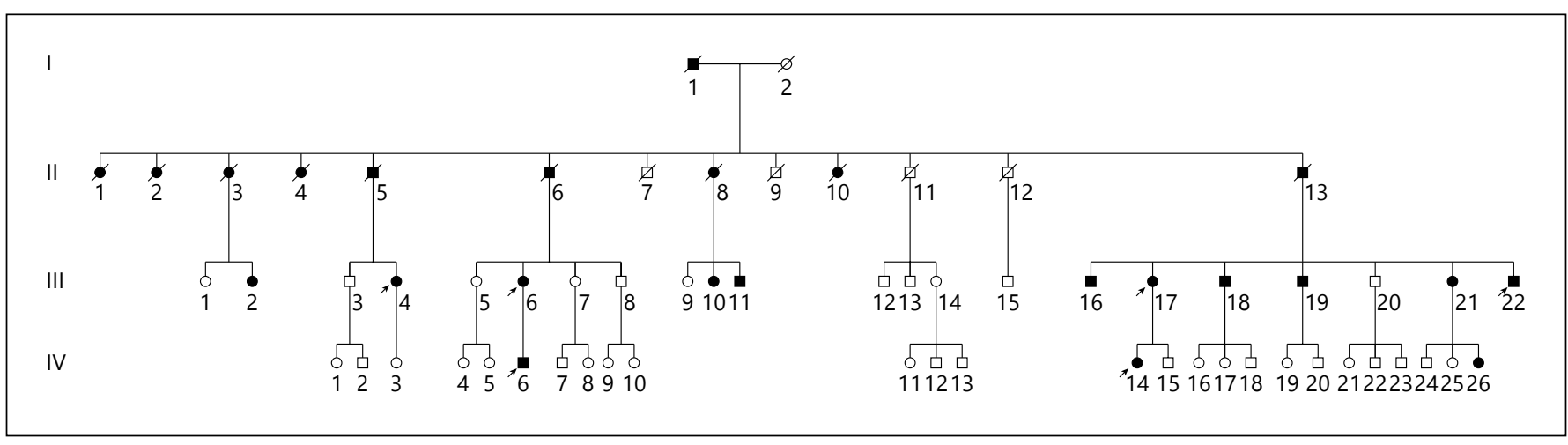

Fig. 1. Pedigree of a large German family segregating autosomal dominant sensorineural hearing loss due to a novel splice site mutation (c.554-1G>A) of the MYO6 gene. Circles represent females and squares males. Filled symbols indicate clinically affected individuals. Participating patients are marked by arrows.

et al., 1996], MYO3A [Dose and Burnside, 2000], MYO7A [Weil et al., 1995] and MYO15A [Wang et al., 1998], MYO6 belongs to the group of unconventional myosins with an indispensable function in the inner ear. Like other myosins, myosin VI consists of a motor domain containing an ATP- and an actin-binding site and a globular tail [Avraham et al., 1997].

For a hearing-impaired individual, an exact diagnosis and adequate auditory rehabilitation is of paramount importance to maintain communication skills and to prevent secondary social problems. In this regard, it has been proposed that knowledge of the underlying molecular aetiology may be crucial for an individualized management using modern auditory rehabilitation techniques. Especially data on the differential prognosis of speech perception after cochlear implantation would be beneficial when counselling affected patients. However, there are just very few studies published which report the clinical details of the outcome after cochlear implantation in patients with different types of hereditary hearing loss thereby limiting the prospects of a phenotype-driven patient management [da Motta et al., 2012; Eppsteiner et al., 2012; Henricson et al., 2012; Lai et al., 2012; Mesolella et al., 2004; Nishizaki et al., 1999; Yamaguchi et al., 1997]. Besides 1 affected patient in a 6-generation MYO6 family who received a cochlear implant [Sanggaard et al., 2008], the literature lacks detailed data on the potential outcome of DFNA22 patients after cochlear implantation. Here, we performed linkage analysis in a large German family with autosomal dominant postlingual non-syndromic sensorineural hearing loss. In addition to the identification and molecular characterization of a novel splice site muta- tion of the MYO6 gene, we present the clinical characteristics and favourable auditory rehabilitation outcome of affected individuals in this family.

\section{Methods}

\section{Patients}

A large German family (fig. 1) with autosomal dominant symmetric sensorineural hearing loss segregating in 4 generations was identified at the Department of Otolaryngology, Head and Neck Surgery of the University of Cologne, Germany (tertiary referral centre). All participating family members gave their written informed consent. The study was approved by the Ethics Committees of the Medical Faculties of the University of Cologne and the University of Ulm, Germany. All investigations have been conducted according to the principles expressed in the Declaration of Helsinki.

\section{Clinical Evaluation}

We used subjective audiometry including assessment of puretone audiometry, tympanometry and transient-evoked otoacoustic emission measurement (ILO 88/ILO 92 system, EST! Medizintechnik AG, Germany) as standard audiological test battery for all participating patients. Additionally, stapedial reflexes and speech audiometry by means of the Freiburger monosyllable word test were evaluated. Speech understanding was tested using the adaptive Oldenburger sentence test in background noise and in quiet. For auditory brainstem response measurement, we used alternating click polarity presented via tubal insert phones as a standard recording procedure (Nicolet Bravo NT, Viasys Healthcare Inc., USA). Threshold audiograms were obtained after otoscopic examination with pure-tone audiometry in a sound-treated room according to current clinical standards. We used air and bone conduction at $125,250,500,1000,2000,4000$, and $8000 \mathrm{~Hz}$ for all affected participants. The neuro-otological assessment was completed using measures of vestibular and balance function. Here, the caloric testing of vestibular function as well as the evaluation of postural control using the Balance Master (NeuroCom, Clackamas, Oreg., USA) were performed. 
A standard 12-lead electrocardiogram (Schiller AT-10 plus, Essen, Germany) was recorded in 1 patient and interpreted independently by 2 experienced cardiologists. Additionally, transthoracic echocardiography was performed in the same patient using the Philips iE33 system (Hamburg, Germany) equipped with a $5-\mathrm{MHz}$ phased array transducer. Standard views in the parasternal long and short axis and apical 4-, 2- and 3-chamber views were recorded according to current guidelines [Cheitlin et al., 2003; Lang et al., 2005]. Global left ventricular function was assessed by measuring the left ventricular ejection fraction applying Simpson's method.

\section{Molecular Investigations}

Genomic DNA was extracted from peripheral blood lymphocytes according to standard procedures. For linkage analysis, DNA of 6 participating affected family members was subjected to a genome-wide scan using the Genome-Wide Human SNP Array 6.0 (Affymetrix, Santa Clara, Calif., USA). Linkage analysis was performed assuming autosomal dominant inheritance, full penetrance, and a disease gene frequency of 0.0001 . Multipoint logarithm of odds (LOD) scores were calculated using the program ALLEGRO [Gudbjartsson et al., 2000]. All data handling was done using the graphical user interface ALOHOMORA [Ruschendorf and Nurnberg, 2005]. The coding exons of MYO6 were analysed by PCR amplification on genomic DNA with subsequent direct sequencing of PCR products (details on PCR conditions and intronic oligonucleotide primers are available upon request). Sequence analysis was performed on an ABI PRISM 3730 DNA analyser with BigDye chemistry v3.1 (Applied Biosystems). All exons and flanking intronic sequences were compared to the MYO6 reference sequence (GenBank entry NM_004999). For the detected mutation, 90 healthy controls of Caucasian ethnicity were screened. Additionally, the Exome Variant Server was checked for the alteration (snp.gs.washington.edu/EVS). For splice prediction, the program NNSplice (http://www.fruitfly.org/seq_tools/splice.html) was used. RNA from whole blood was isolated using the PAXgene ${ }^{\mathrm{TM}}$ Blood RNA Kit (Qiagen, Hilden, Germany). cDNA was synthesized using the One-Step RT-PCR kit (Qiagen) according to the manufacturer's instructions. Subcloning was performed using the TOPO TA Cloning Kit (Invitrogen ${ }^{\circledR}$ ).

\section{Results}

\section{Genetic Findings}

Genome-wide linkage analysis in 6 affected family members (III.4, III.6, III.17, III.22, IV.6 and IV.14; fig. 1) identified a putative linkage to the chromosomal regions 3 p11.1-q13.31 (defining a critical region of $27.4 \mathrm{Mb}$ between markers rs35401223 and rs7650115), 20q13.33 (2.57 Mb between markers rs2386937 and rs11907696) and 6p21.1-q22 (75.0 Mb between markers rs1340862 and rs11966892; fig. 2a). The multipoint LOD score of each chromosomal region was 2.11 , which is the maximum expected LOD score from simulation analysis and thus showed perfect cosegregation of marker alleles/haplotypes with the disease. The only gene known to be associated with autosomal dominant non-syndromic hearing loss within the putative linkage intervals was myosin VI (MYO6). We therefore analysed all 34 coding exons and adjacent splice sites of MYO6 by PCR amplification on blood-derived genomic DNA and subsequent direct sequencing of PCR products in patient IV.14. We identified a novel splice site mutation (c.554-1G $>$ A) at the invariant -1 position of the consensus splice acceptor site of exon 7 of MYO6 (fig. 2b). The mutation cosegregates with the phenotype in the participating individuals, was not detected in 180 control chromosomes and is not listed in the Exome Variant Server, which is covering around 8600 chromosomes of European ancestry at this position. In silico splice site analysis predicted a deleterious effect of the mutation on splicing efficacy ( 0.99 for the wild-type vs. unrecognizable for the mutation at a minimum score of 0.1 ) and the nucleotide exchange thus probably leads to the loss of the splice acceptor site of exon 7. In patient IV.14, different alternative splice products of MYO6 were identified by cDNA analysis with reverse transcription PCR, subsequent subcloning and sequencing. Out of the 4 identified altered splice products, 1 will lead to an inframe deletion (p.Ala185_Leu189del) while the others will cause truncated proteins (p.Ala185Glufs*17, p.Ala185Glyfs*16, p.Ala185Valfs*8; see schematic overview in fig. 3 ).

\section{Clinical Findings}

The family included 24 affected individuals in 4 generations and audiograms were obtained from 6 affected family members (fig. 1). Except for their hearing impairment, all patients had a normal otorhinolaryngology status and a negative history of major neurologic disorders. The hearing loss was sensorineural, bilateral and by history progressive in all participating family members. At onset, the hearing impairment was detected at the midand high frequencies, degrading to a profound hearing impairment involving all frequencies. The hearing impairment was first noticeable at school age. However, audiological examinations were available from adulthood only, due to considerable delay of the diagnosis until hearing aids were required. In the youngest affected participating family member (IV.6, 26 years of age), sensorineural hearing loss started from $20 \mathrm{~dB} \mathrm{HL}$ at $500 \mathrm{~Hz}$ and affected the mid-frequencies and high frequencies (1000$8000 \mathrm{~Hz}$ ) with $40-55 \mathrm{~dB} \mathrm{HL}$ with a peak at $1500 \mathrm{~Hz}$. The subjectively progressive nature of the hearing impairment is consistently reported by all participating family 
Fig. 2. Linkage analysis results and sequencing chromatogram. a Genomewide graphical view of LOD scores using SNP array mapping in 6 affected individuals (marked by arrows in fig. 1). Putative linkage was found for the chromosomal regions 3p11.1-q13.31, 20q13.33 and 6p21.1-q22.1. b Electropherogram of patient IV.14 showing a novel heterozygous splice site mutation c.554-1G $>$ A (arrow) at the splice acceptor site of exon 7 of the MYO6 gene.

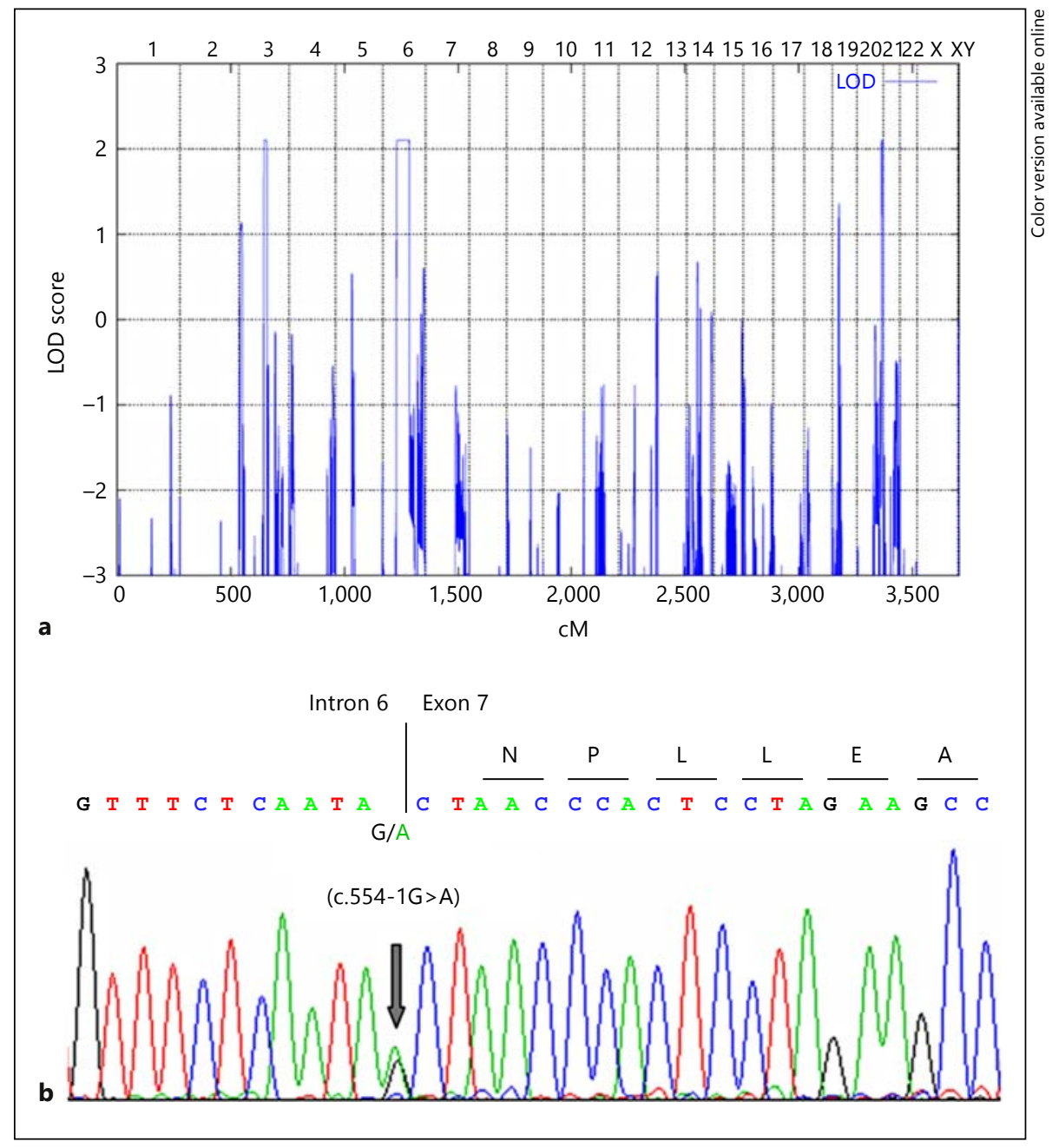

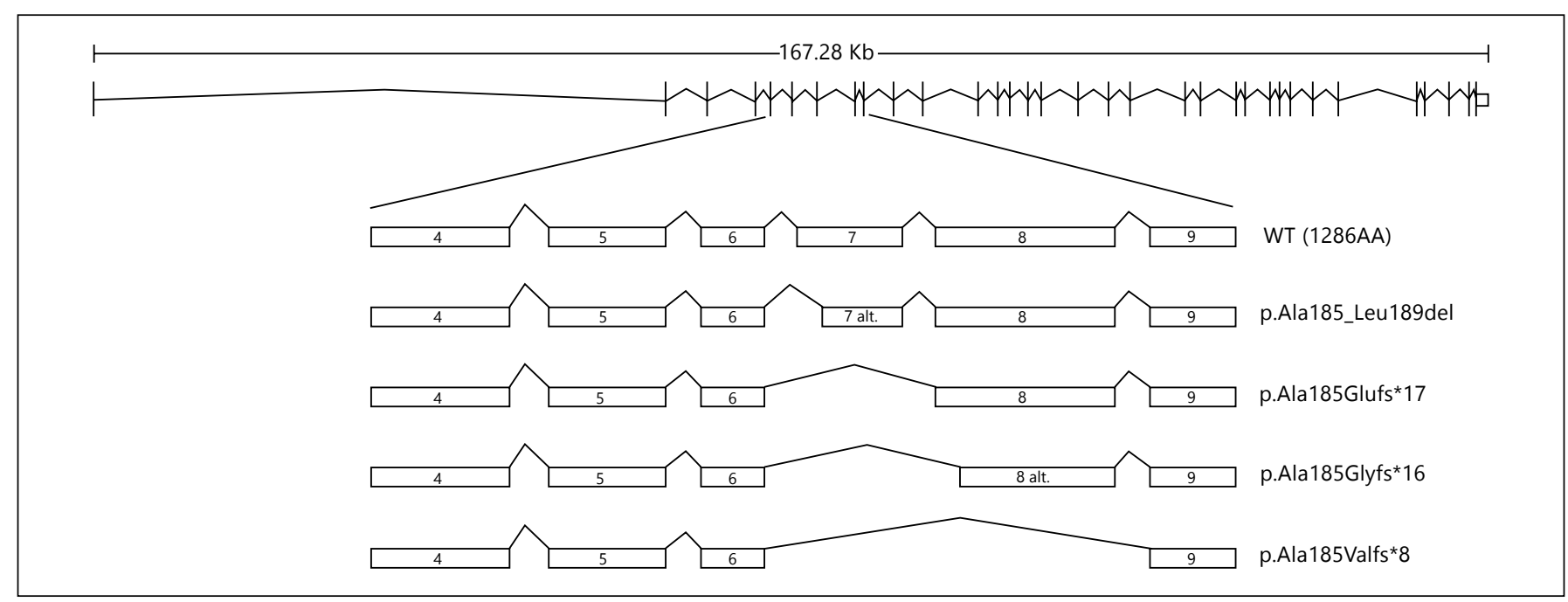

Fig. 3. Schematic overview of the consequences of the identified mutation on MYO6 splicing behaviour. cDNA analysis by PCR amplification, subcloning and subsequent sequencing revealed several altered, deleterious transcripts in the presence of the c.5541G>A mutation in MYO6. 
Table 1. Pre- and postoperative audiological evaluation of both implantees

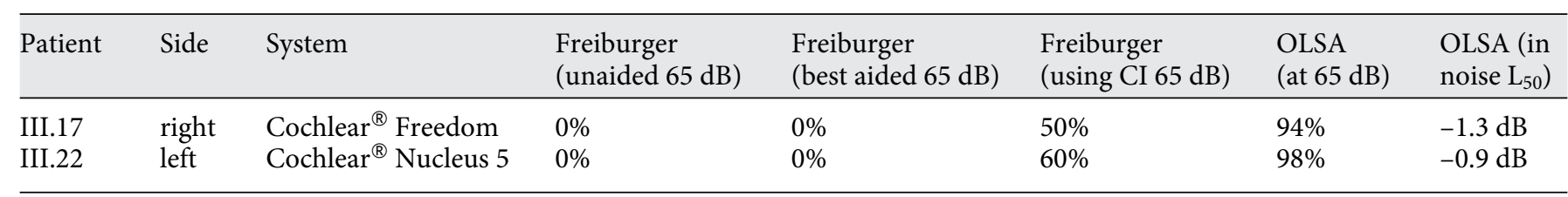

Freiburger $=$ Freiburger monosyllable word test; $\mathrm{CI}=$ cochlear implant; OLSA $=$ Oldenburger sentence test.

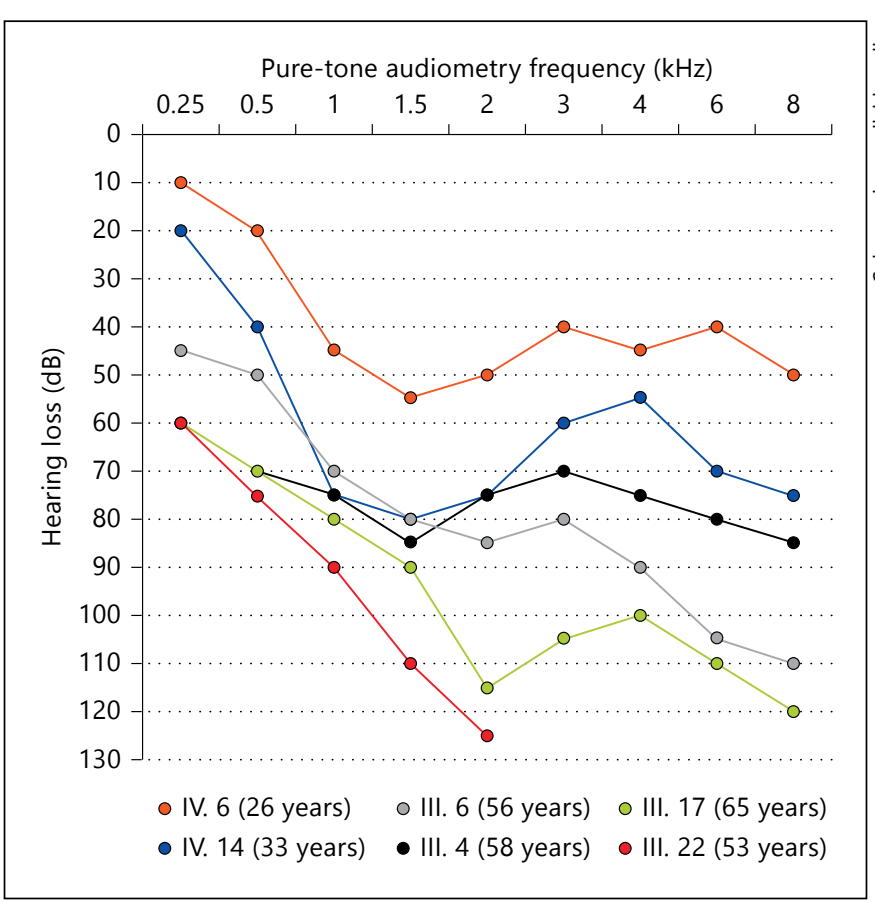

Fig. 4. Overlayed pure-tone audiograms of the different participating family members. All patients had a steeply sloping, flat audiogram.

members and can also be indirectly deduced from overlaying their pure-tone audiograms performed at different ages (fig. 4). All participating individuals had a sensorineural hearing loss, typically with a steeply sloping, flat audiogram. A correlation between age and severity of sensorineural hearing loss can be observed leading to a profound hearing loss with only residual hearing detectable up to $2000 \mathrm{~Hz}$ at the age of 65 . Conventional air conduction hearing aids were prescribed around the age of 20 in family members III.4, III.17, III.22 and IV.6. However, due to limitations of conventional amplification in profound hearing impairment, 2 family members (III.17 and III.22) became candidates for cochlear implantation at the age of 65 and 53, respectively. Both patients were implanted using the typical cochlear implant round window route, following a transmastoid facial recess approach. The full insertion of a contoured electrode array of the Nucleus freedom (III.17) and the Nucleus 5 (III.22) (Cochlear Corporation, Australia) was uneventful. Postoperatively, aided thresholds as well as speech recognition using the Freiburger monosyllable word test were in line with the subjective favourable outcome and performance of the patients. Furthermore, the adaptive Oldenburger sentence test in background noise and in quiet showed good functional results (table 1). There were no subjective and objective vestibular dysfunctions as well as retrocochlear pathology in any of the participating family members. Patient IV.14 underwent detailed cardiac evaluation after the identification of the mutation in the MYO6 gene as associated abnormalities had been reported in the literature [Mohiddin et al., 2004]. However, we did not find signs of cardiomyopathy by history or by clinical, electrocardiogram or echocardiographic examinations in this patient. No cardiac diseases were reported in any of the other participating patients nor did any of the patients report on a retinal disease.

\section{Discussion}

In this study, we linked autosomal dominant hearing loss in an extended German family to a region on chromosome 6 overlapping with the DFNA22/DFNB37 locus. Indeed, we could identify a novel MYO6 mutation (c.554$1 \mathrm{G}>\mathrm{A}$ ) at the splice acceptor site of exon 7 in all affected participating family members. Subsequent cDNA analysis revealed different aberrantly spliced mRNAs predicted to lead to an in-frame deleted or a truncated protein thereby - and together with the absence of the alteration in a large number of controls - confirming the pathogenic nature of the identified splice site mutation. To date, only just 7 families with mutations in MYO6 have been 
identified. Depending on the kind of alteration, MYO6 mutations have been found in either autosomal dominant hearing loss type DFNA22 [Hilgert et al., 2008; Melchionda et al., 2001; Sanggaard et al., 2008; Topsakal et al., 2010] or autosomal recessive hearing loss type DFNB37 [Ahmed et al., 2003]. Moreover, mutations in the murine ortholog can result in autosomal recessive deafness in Snell's waltzer [Avraham et al., 1995] or autosomal dominant deafness in Tailchaser mice [Hertzano et al., 2008]. In DFNA22, 2 different missense mutations [Melchionda et al., 2001; Mohiddin et al., 2004], 1 splice site alteration [Hilgert et al., 2008] and 1 nonsense mutation [Sanggaard et al., 2008] have been reported. Concerning a putative genotype-phenotype correlation, it was suggested that an intact motor domain is associated with a less severe phenotype. For the missense mutations, a dominant negative effect was hypothesized, whereas for the nonsense and splice site mutation, also a deleterious effect on dimerization or a nonsense-mediated mRNA decay resulting in decreased protein levels were discussed [Topsakal et al., 2010]. The mutation identified in this report does not lead to complete degradation by nonsense-mediated mRNA decay (at least in blood cells) as cDNA analysis in 1 of the patients revealed the expression of normal (wildtype) and mutant transcripts. The corresponding proteins are predicted to lack parts of the motor domain (at least 5 amino acids), the complete coiled coil domain, and the globular tail so that a dominant negative effect, which would be dependent on a dimerization of mutated and wild-type proteins, seems to be rather improbable at least for the truncated proteins. Therefore, our findings are better compatible with haploinsufficiency as the causative pathomechanism. Only for the in-frame deletion could a dominant negative effect resulting from the deletion of these 5 highly conserved amino acids be discussed. Additionally, our results support earlier studies which showed a more severe phenotype in families with mutations within the motor domain [Topsakal et al., 2010]. The hearing impairment in the participating affected individuals was found to be sensorineural, and characterized by a postlingual symmetrical childhood onset. Over decades, this hearing disorder progresses slowly leading to profound hearing impairment and deafness. This is in line with earlier studies on the clinical phenotype of autosomal dominant MYO6-associated hearing loss [Hilgert et al., 2008; Melchionda et al., 2001; Mohiddin et al., 2004; Sanggaard et al., 2008]. Unfortunately, only 6 affected family members in the age range of 26-65 years participated and issues about the age of onset and possibly reduced penetrance could therefore not be investi-

Cochlear Implantation in Hearing Loss

Type DFNA22 gated. In contrast to earlier studies for other MYO6 mutations [Ahmed et al., 2003; Mohiddin et al., 2004], the mutation identified in this study seems according to the clinical history of the participating family members and detailed cardiac evaluation in 1 affected individual (IV.14) not to be associated with visual, vestibular or cardiac dysfunction.

Sensorineural hearing impairment showing insufficient benefits from amplification by conventional hearing aids represents a clinical indication for cochlear implantation. Bypassing the sensory organ of the inner ear, cochlear implants directly activate auditory nerve fibres, transmit auditory signals through the central neural pathway and ultimately yield speech understanding in the cortex. The performance outcome with cochlear implants, however, varies significantly among implantees probably because of the extremely heterogeneous aetiology of sensorineural hearing loss. As cochlea implantation is an invasive and costly procedure, identification of prognostic factors is of importance and it has been proposed that the elucidation of the underlying genetic cause of the disease might in the future be helpful to better predict the outcome of cochlear implantation in hereditary hearing impairment [Eppsteiner et al., 2012]. Myosin VI has been reported to have a role in both endocytic trafficking [Aschenbrenner et al., 2003] and actin-based intracellular transport of vesicles and organelles. It is highly expressed in the cuticular plate and pericuticular necklace of auditory hair cells [Avraham et al., 1997] and mutations in orthologs of myosin VI in different species have been associated with disorganized hair bundles and an impaired mechanotransduction [Hertzano et al., 2008; Kappler et al., 2004]. Moreover, data by Roux et al. [2009] provide evidence that myosin VI is also involved in the morphological and functional maturation of inner hair cell ribbon synapses. Additionally, myosin VI is required for efficient calcium-dependent exocytosis of synaptic vesicles in inner hair cell ribbon synapses [Roux et al., 2009]. Taken together, myosin VI seems to be mainly involved in the maintenance and proper function of sensory hair cells. As DFNA22 mutations thus most probably affect the function of the hair cells, cochlear implants that bypass the hair cells and stimulate the auditory nerve fibres could be expected to represent a rehabilitation option with good prognosis. Indeed, the auditory performance of the 2 implantees demonstrates that this theoretical expectation could be clinically validated and postimplantation auditory test results showed a clear benefit of speech recognition in quiet and noise. To date, only very few reports on the outcome of cochlear implantation in patients with defined types of hereditary hearing loss have been published 
[da Motta et al., 2012; Henricson et al., 2012; Lai et al., 2012; Mesolella et al., 2004; Nishizaki et al., 1999; Sanggaard et al., 2008; Yamaguchi et al., 1997]. All of these describe a favourable outcome after cochlear implantation; however, it remains unclear whether this demonstrates a generally good response of this therapy in hereditary hearing loss or rather a publication bias. Further comprehensive and larger studies should thus focus on a potential correlation between a specific genotype and clinical rehabilitation by cochlear implantation.

In conclusion, we linked autosomal dominant hearing loss in a German family to the DFNA22/DFNB37 locus, and identified a novel mutation in myosin VI that cosegregated with the hearing loss in the family. Furthermore, we could demonstrate that patients exhibiting hearing impairment due to MYO6 dysfunction may represent good candidates for cochlear implantation.

\section{Acknowledgements}

We thank the family members for participation and Katrin Friedrich and Ingrid Goebel for excellent technical assistance. This work was funded by European Commission FP6 Integrated Project EUROHEAR, Grant LSHG-CT-20054512063 to C.K. The authors would like to thank the NHLBI GO Exome Sequencing Project and its ongoing studies which produced and provided exome variant calls for comparison: the Lung GO Sequencing Project (HL-102923), the WHI Sequencing Project (HL-102924), the Broad GO Sequencing Project (HL-102925), the Seattle GO Sequencing Project (HL-102926) and the Heart GO Sequencing Project (HL-103010).

\section{Disclosure Statement}

The authors declare that they have no financial conflict of interest.

\section{References}

Ahmed ZM, Morell RJ, Riazuddin S, Gropman A, Shaukat S, Ahmad MM, Mohiddin SA, Fananapazir L, Caruso RC, Husnain T, Khan SN, Griffith AJ, Friedman TB, Wilcox ER: Mutations of MYO6 are associated with recessive deafness, DFNB37. Am J Hum Genet 2003;72:1315-1322.

Aschenbrenner L, Lee T, Hasson T: Myo6 facilitates the translocation of endocytic vesicles from cell peripheries. Mol Biol Cell 2003;14: 2728-2743.

-Avraham KB, Hasson T, Sobe T, Balsara B, Testa JR, Skvorak AB, Morton CC, Copeland NG, Jenkins NA: Characterization of unconventional MYO6, the human homologue of the gene responsible for deafness in Snell's waltzer mice. Hum Mol Genet 1997;6:1225-1231.

Avraham KB, Hasson T, Steel KP, Kingsley DM, Russell LB, Mooseker MS, Copeland NG, Jenkins NA: The mouse Snell's waltzer deafness gene encodes an unconventional myosin required for structural integrity of inner ear hair cells. Nat Genet 1995;11:369-375.

-Cheitlin MD, Armstrong WF, Aurigemma GP, Beller GA, Bierman FZ, Davis JL, Douglas PS, Faxon DP, Gillam LD, Kimball TR, Kussmaul WG, Pearlman AS, Philbrick JT, Rakowski H, Thys DM, Antman EM, Smith SC Jr, Alpert JS, Gregoratos G, Anderson JL, Hiratzka LF, Hunt SA, Fuster V, Jacobs AK, Gibbons RJ, Russell RO: ACC/AHA/ASE 2003 Guideline Update for the Clinical Application of Echocardiography: summary article. A report of the American College of Cardiology/American Heart Association Task Force on Practice Guidelines (ACC/AHA/ASE Committee to Update the 1997 Guidelines for the Clinical
Application of Echocardiography). Circulation 2003; 108:1146-1162.

da Motta LH, Felix TM, de Souza LT, LavinskyWolff M, Costa-Motta FM, de Faria MR, Lavinsky L: Prevalence of the $35 \mathrm{delG}$ mutation in deaf south brazilian infants submitted to cochlear implantation. Int J Pediatr Otorhinolaryngol 2012;76:287-290.

Dose AC, Burnside B: Cloning and chromosomal localization of a human class III myosin. Genomics 2000;67:333-342.

Eppsteiner RW, Shearer AE, Hildebrand MS, Deluca AP, Ji H, Dunn CC, Black-Ziegelbein EA, Casavant TL, Braun TA, Scheetz TE, Scherer SE, Hansen MR, Gantz BJ, Smith RJ: Prediction of cochlear implant performance by genetic mutation: the spiral ganglion hypothesis. Hear Res 2012;292:51-58.

-Gudbjartsson DF, Jonasson K, Frigge ML, Kong A: Allegro, a new computer program for multipoint linkage analysis. Nat Genet 2000;25: $12-13$.

-Hasson T, Skowron JF, Gilbert DJ, Avraham KB, Perry WL, Bement WM, Anderson BL, Sherr EH, Chen ZY, Greene LA, Ward DC, Corey DP, Mooseker MS, Copeland NG, Jenkins NA: Mapping of unconventional myosins in mouse and human. Genomics 1996;36:431439.

Henricson C, Wass M, Lidestam B, Moller C, Lyxell B: Cognitive skills in children with usher syndrome type 1 and cochlear implants. Int J Pediatr Otorhinolaryngol 2012;76:1449-1457.

Hertzano R, Shalit E, Rzadzinska AK, Dror AA, Song L, Ron U, Tan JT, Shitrit AS, Fuchs H, Hasson T, Ben-Tal N, Sweeney HL, de Angelis MH, Steel KP, Avraham KB: A Myo6 muta- tion destroys coordination between the myosin heads, revealing new functions of myosin vi in the stereocilia of mammalian inner ear hair cells. PLoS Genet 2008;4:e1000207.

Hilgert N, Smith RJ, Van Camp G: Forty-six genes causing nonsyndromic hearing impairment: which ones should be analyzed in DNA diagnostics? Mutat Res 2009;681:189-196.

Hilgert N, Topsakal V, van Dinther J, Offeciers E, Van de Heyning P, Van Camp G: A splice-site mutation and overexpression of Myo6 cause a similar phenotype in two families with autosomal dominant hearing loss. Eur J Hum Genet 2008;16:593-602.

Kappler JA, Starr CJ, Chan DK, Kollmar R, Hudspeth AJ: A nonsense mutation in the gene encoding a zebrafish myosin VI isoform causes defects in hair-cell mechanotransduction. Proc Natl Acad Sci USA 2004;101:13056-13061.

Lai R, Hu P, Zhu F, Zhu G, Vivero R, Peng A, Wu W, Xiao Z, Liu X, Xie D: Genetic diagnosis and cochlear implantation for patients with nonsyndromic hearing loss and enlarged vestibular aqueduct. J Laryngol Otol 2012;126: 349-355.

Lang RM, Bierig M, Devereux RB, FlachskampfFA, Foster E, Pellikka PA, Picard MH, Roman MJ, Seward J, Shanewise JS, Solomon SD, Spencer KT, Sutton MS, Stewart WJ: Recommendations for chamber quantification: a report from the American Society of Echocardiography's Guidelines and Standards Committee and the Chamber Quantification Writing Group, developed in conjunction with the European Association of Echocardiography, a branch of the European Society of Cardiology. J Am Soc Echocardiogr 2005;18:1440-1463. 
Melchionda S, Ahituv N, Bisceglia L, Sobe T, Glaser F, Rabionet R, Arbones ML, Notarangelo A, Di Iorio E, Carella M, Zelante L, Estivill X, Avraham KB, Gasparini P: Myo6, the human homologue of the gene responsible for deafness in Snell's waltzer mice, is mutated in autosomal dominant nonsyndromic hearing loss. Am J Hum Genet 2001;69:635-640.

-Mesolella M, Tranchino G, Nardone M, Motta S, Galli V: Connexin 26 mutations in nonsyndromic autosomal recessive hearing loss: speech and hearing rehabilitation. Int J Pediatr Otorhinolaryngol 2004;68:995-1005.

Mohiddin SA, Ahmed ZM, Griffith AJ, Tripodi D, Friedman TB, Fananapazir L, Morell RJ: Novel association of hypertrophic cardiomyopathy, sensorineural deafness, and a mutation in unconventional myosin VI (MYO6). J Med Genet 2004;41:309-314.
Nishizaki K, Fukushiama K, Oda Y, Masuda A, Hayashi S, Nagayasu N, Yoshino T, Kashihara K, Takahashi K, Masuda Y: Cochlear implantation for symptomatic hereditary deafness. Acta Otolaryngol Suppl 1999;540:34-37.

- Roux I, Hosie S, Johnson SL, Bahloul A, Cayet N, Nouaille S, Kros CJ, Petit C, Safieddine S: Myosin VI is required for the proper maturation and function of inner hair cell ribbon synapses. Hum Mol Genet 2009;18:4615-4628.

Ruschendorf F, Nurnberg P: ALOHOMORA: a tool for linkage analysis using 10K SNP array data. Bioinformatics 2005;21:2123-2125.

anggaard KM, Kjaer KW, Eiberg H, Nurnberg G, Nurnberg P, Hoffman K, Jensen H, Sorum C, Rendtorff ND, Tranebjaerg L: A novel nonsense mutation in MYO6 is associated with progressive nonsyndromic hearing loss in a Danish DFNA22 family. Am J Med Genet A 2008;146A:1017-1025.

- Topsakal V, Hilgert N, van Dinther J, Tranebjaerg L, Rendtorff ND, Zarowski A, Offeciers E, Van Camp G, van de Heyning P: Genotypephenotype correlation for DFNA22: characterization of non-syndromic, autosomal dominant, progressive sensorineural hearing loss due to MYO6 mutations. Audiol Neurootol 2010;15:211-220.
Wang A, Liang Y, Fridell RA, Probst FJ, Wilcox ER, Touchman JW, Morton CC, Morell RJ, Noben-Trauth K, Camper SA, Friedman TB: Association of unconventional myosin MYO15 mutations with human nonsyndromic deafness DFNB3. Science 1998;280: 1447-1451.

Weil D, Blanchard S, Kaplan J, Guilford P, Gibson F, Walsh J, Mburu P, Varela A, Levilliers J, Weston MD, et al: Defective myosin VIIA gene responsible for Usher syndrome type 1B. Nature 1995;374:60-61.

Yamaguchi T, Himi T, Harabuchi Y, Hamamoto M, Kataura A: Cochlear implantation in a patient with mitochondrial disease - KearnsSayre syndrome: a case report. Adv Otorhinolaryngol 1997;52:321-323. 